\title{
The Effect of Ultrasonic Treatment on Thermal Stability of the Cured Epoxy/Layered Silicate Nanocomposite
}

\author{
N. Y. Yuhana, ${ }^{1}$ S. Ahmad, ${ }^{2}$ and A. R. Shamsul Bahri ${ }^{3}$ \\ ${ }^{1}$ Department of Chemical and Process Engineering, Universiti Kebangsaan Malaysia, Selangor, 43000 Bangi, Malaysia \\ ${ }^{2}$ Department of Material Science, Universiti Kebangsaan Malaysia, Selangor, 43000 Bangi, Malaysia \\ ${ }^{3}$ Crop Improvement and Protection Unit, Rubber Research Institute-Experimental Station, \\ 47000 Sungai Buloh, Malaysia \\ Correspondence should be addressed to N. Y. Yuhana, yuliana@eng.ukm.my
}

Received 6 September 2011; Revised 20 January 2012; Accepted 20 January 2012

Academic Editor: Wen-Hua Sun

Copyright (๑) 2012 N. Y. Yuhana et al. This is an open access article distributed under the Creative Commons Attribution License, which permits unrestricted use, distribution, and reproduction in any medium, provided the original work is properly cited.

The effect of ultrasonic treatment on thermal stability of binary systems containing epoxy and organic chemically modified montmorillonite (Cloisite 30B) was studied. Differential scanning calorimetry (DSC), thermal gravimetric analysis (TGA), transmission electron microscopy (TEM), and wide angle X-ray diffraction (WAXD) analysis were utilized. The mixing of epoxy and Cloisite 30B nanocomposites was performed by mechanical stirring, followed by 1 or 3-hour ultrasonic treatment, and polyetheramine as the curing agent. Both XRD and TEM analyses confirmed that the intercalation of Cloisite 30B was achieved. The $d_{0}$ spacings for silicate in cured sample prepared at 1- and 3-hour duration of ultrasonic treatment were about 21 and $18 \AA$, respectively. This shows that shorter duration or ultrasonic treatment may be preferable to achieve higher $d_{0}$ spacing of clay. This may be attributed to the increase in viscosity as homopolymerization process occurred, which restricts silicate dispersion. The 1hour sonicated samples seem to be more thermally stable during the glass transition, but less stable during thermal decomposition process.

\section{Introduction}

The use of ultrasonic treatment was proven to be feasible in mixing nanoclay in epoxy. The breakup of clay agglomerates can be assisted by the vibration of ultrasonic wave [1-3]. However, the longer time of ultrasonic treatment may lead to early polymerization and may degrade the properties of nanocomposite due to increased temperature during sonication. The other problem is formation of bubbles in liquid mixture, hence leaving voids in hardened epoxy samples $[3,4]$. The size of voids became larger as sonication time increased. Bernard et al. also reported the increase in curing rate for ultrasonically-processed samples.

Epoxy/layered silicate nanocomposites exhibit improved thermal, barrier, and mechanical properties [5].

It is often stated that the layered silicate can increase the glass transition temperature $\left(T_{g}\right)$ by restricting the epoxy chain mobility. They can also reduce the $T_{g}$, due to the presence of hydroxyl group in unmodified mica and acidic onium ion in organically modified silicate, which both are capable of acting as a catalyst for epoxy homopolymerization [6, 7]. If this happens, the unreacted curing agent plasticizes the matrix. Kinloch and Taylor concluded that intercalated structure of silicate can improve the $T_{g}[8]$. Becker et al. found that increasing organoclay concentration is steadily decreased $T_{g}$ [6].

The improvement in thermal stability of nanocomposites is often mentioned to be attributable to the torturous path for volatile decomposition products. The thermal stability of epoxy increased with the contents of inorganic components, because of the char yield increases [9]. The char layer can protect unburned structure materials during fire. The decrease in thermal stability may be ascribable to the catalytic activity of organoclay on epoxy decomposition [2].

In this paper, we report the morphology and thermal stability of epoxy clay nanocomposites prepared by the ultrasonic process. 


\section{Experimental}

\subsection{Materials}

Diglycidyl ether bisphenol A (DGEBA) epoxy resin, Epikote 828, was obtained from Hexion Specialty Chemicals, Korea. The epoxide equivalent weight was $187 \mathrm{~g} / \mathrm{mol}$. The liquid curing agent, Baxxodur TM EC301 (formerly known as Polyetheramine D230), was supplied by BASF. The composition is alpha-(2-Aminomethylethyl)-omega-(2-aminomethylethoxy)-poly(oxy(methyl-1,2-ethanediyl)). PMMA-grafted natural rubber Megatex 30 was gained from Green HPSP (M) Sdn. Bhd. The organically modified montmorillonite used was Cloisite 30B, purchased from Southern Clay Products, USA. The composition of the modifier is bis (hydroxyl ethyl) methyl tallow alkyl ammonium salts with montmorillonite.

2.2. Sample Preparation by Mechanical Stirring and Ultrasonic Treatment. One hundred grams of bisphenol A diglycidyl ether were added to a stainless steel cup. It was heated to about $55^{\circ} \mathrm{C}$ by using a hot plate. This was followed by the addition of 5 phr Cloisite $30 \mathrm{~B}$ and mechanical stirring at about $50 \mathrm{rpm}$ for about $1 \mathrm{hr}$. Next, the cup was immersed in ultrasonic bath, Powersonic UB 410, set at medium amplitude and $50^{\circ} \mathrm{C}$ for 1 or 3 hours. Then, a stoichiometric amount of the liquid curing agent was added and stirred for about 10 minutes. Finally, the mixture was cured at $40^{\circ} \mathrm{C}$ for 20 hours and postcuring was done at $125^{\circ} \mathrm{C}$ for 3 hours, in the convection oven.

2.3. Transmission Electron Microscopy. The samples containing rubber were stained with osmium tetroxide for $1 \mathrm{hr}$ before cutting with a Reichert Ultracut E ultramicrotome, utilizing a glass knife at room temperature. The 70-90 nm thick samples were collected on hexagonal 200 mesh copper grids, and the micrographs were obtained from Philips TEM model CM12 with Soft Imaging Viewer software.

2.4. X-Ray Diffraction. To study the dispersion of Cloisite 30B inside the epoxy, X-ray diffraction was conducted at room temperature, using a Bruker AXS D8 Advance Xray diffractometer. The X-ray beam was copper-filtered and radiation generated at operating conditions of $40 \mathrm{kV}$ and $40 \mathrm{~mA}$. The X-ray data were obtained from $2.3^{\circ}$ to $10^{\circ}(2 \theta)$ at a scan rate of $0.025^{\circ} / \mathrm{min}$. Thin specimens, about $1 \mathrm{~mm}$ thick, were analyzed. Cloisite $30 \mathrm{~B}$ was also characterized to measure the initial $d$-spacing. The graphs were analyzed using Diffrac Plus (Release 2005) and Eva Version 11 Rev. 0, supplied by Bruker Advance X-ray Solutions.

2.5. Differential Scanning Calorimetry Analysis. The glass transition temperature of cured samples was determined by using Mettler Toledo DSC $822^{\mathrm{e}}$. The sample was heated at $10^{\circ} \mathrm{C} \mathrm{m^{-1 }}$ from 25 to $170^{\circ} \mathrm{C}$. The sample was maintained at that temperature for approximately $2 \mathrm{~min}$, then it was cooled to $-20^{\circ} \mathrm{C}$ at the rate of $20^{\circ} \mathrm{C} \mathrm{min}^{-1}$ to remove any thermal history. The sample was finally rescanned using a modulated heating programme as described above, twice.
2.6. Thermalgravimetric Analysis. The thermal stability of cured samples was studied by utilizing Netzsch STA 449 F3 Jupiter. The sample was heated from 25 to $800^{\circ} \mathrm{C}$ at a rate of $20^{\circ} \mathrm{C} / \mathrm{min}$, under nitrogen flow.

\section{Results and Discussion}

\subsection{Transmission Electron Microscopy}

Figures 1(a) and 1(b) demonstrates the agglomeration of nanosilicate in the cured sample prepared by mechanical stirring, without ultrasonic treatment. Great improvement was achieved when ultrasonic treatment was introduced. It is obvious that the aggregates sizes of nanoclay grow much smaller with increasing time of ultrasonic treatment, as shown in Figures 1(c)-1(d) and 1(e)-1(f). The images of nanoclay are also clearly observed for sonicated prepared samples. The intercalation structure of nanoclay can be seen in Figures 1(d) and 1(f).

3.2. X-Ray Diffraction. From the previous XRD analysis of epoxy silicate nanocomposite prepared by mechanical stirring only, it is indicated that the interlayer spacing of crystalline structure, $d_{001}$, is $19.18 \AA$ [10].

The results of XRD analysis for cured samples prepared by ultrasonic treatment is given in Figure 2. The $d_{001}$ of the epoxy-nanoclay composites prepared with $1 \mathrm{~h}$ and $3 \mathrm{~h}$ ultrasonic stirring were about $21 \AA$ and $18 \AA$, respectively.

The decrease in $d_{0}$ upon longer duration of ultrasonic treatment may be due to the higher viscosity of the mixture in turn attributable to homopolymerization of epoxy. The extragallery reaction, which refers to polymerization process, tends to force the clay to get closer. Hence, lower $d_{0}$ was obtained for 3-hour sonicated sample.

The slightly higher $d_{0}$ for 1-hour sonicated prepared nanocomposite sample may be attributed to easy separation of clay in less viscous mixture. The longer time of ultrasonic treatment will result in increased viscosity of polymer mixture.

3.3. Differential Scanning Calorimetry. The DSC graph and the analysis of $T_{g}$ during the second heating step is illustrated in Figure 3 and Table 1.

During the first heating process, the 1-hour sonicated sample shows a small exothermic peak around $100^{\circ} \mathrm{C}$. The chemical reactions may occur due to incomplete reactions between epoxy and curing agent during curing process. Furthermore, the exothermic peaks with "stages" can be observed upon cooling to $-20^{\circ} \mathrm{C}$. This indicates the polymorphisms of epoxy clay nanocomposites.

It is obvious that epoxy nanocomposite prepared by 1hour ultrasonic treatment possesses the higher value of $T_{g}$. The lower $T_{g}$ may be related to restricted segmental motions near the interface between the epoxy chain and Cloisite 30B. The presence of more bubbles in 3-hour sonicated processed sample can lessen the good adhesion between clay and epoxy matrix at its interphase.

In literature, $T_{g}$ can be increased by the presence of bulky side groups, polar groups, double bonds, and aromatic 


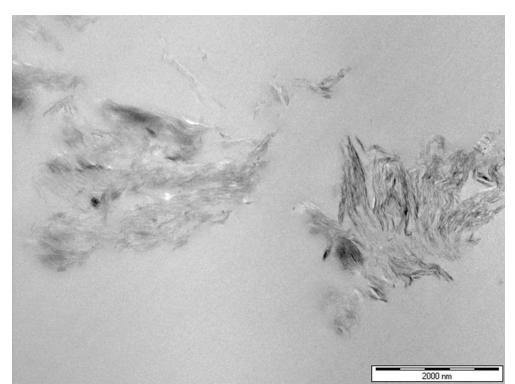

(a)

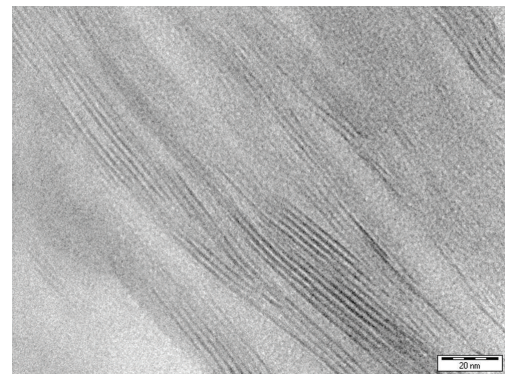

(d)

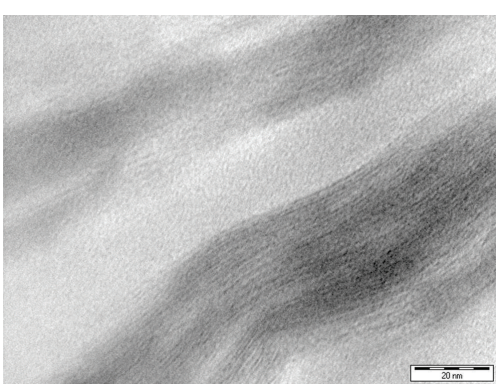

(b)

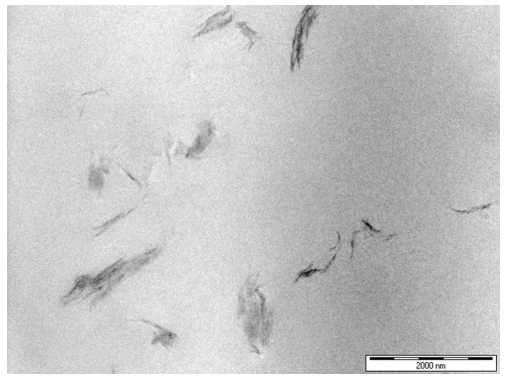

(e)

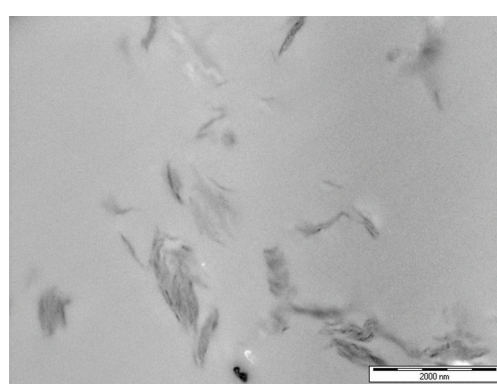

(c)

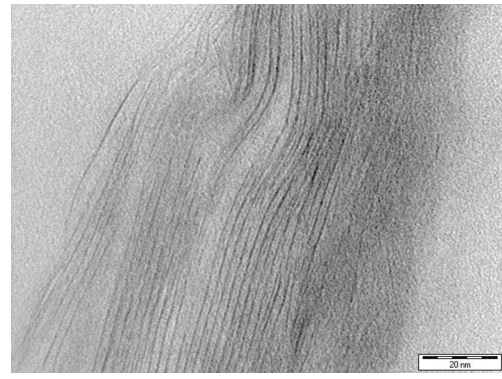

(f)

FIGURE 1: The TEM images of cured samples prepared by ( $\mathrm{a}$ and $\mathrm{b}$ ) mechanical stirring only, where aggregates of silicate can be seen [10] (c and d) 1-hour, (e and f) 3-hour ultrasonic treatment.

TABLE 1: The analysis of glass transition temperatures during the second heating process.

\begin{tabular}{lccc}
\hline Sample & $\begin{array}{c}\text { Onset of glass transition } \\
\text { temperature, } T_{g}\left({ }^{\circ} \mathrm{C}\right)\end{array}$ & $\begin{array}{c}\text { Inflection slope of glass } \\
\text { transition, } m_{g} \\
(\mathrm{~mW} / \mathrm{min})\end{array}$ & $\begin{array}{c}\text { The specific heat } \\
\text { increment, } \Delta c_{p g} \text { alass } \\
\text { transition }(\mathrm{J} / \mathrm{gK})\end{array}$ \\
\hline Ep 530B US $1 \mathrm{hr}$ & 85.03 & 0.69 & 0.393 \\
Ep 530B US 3 hr & 82.57 & 0.78 & 0.439 \\
\hline
\end{tabular}

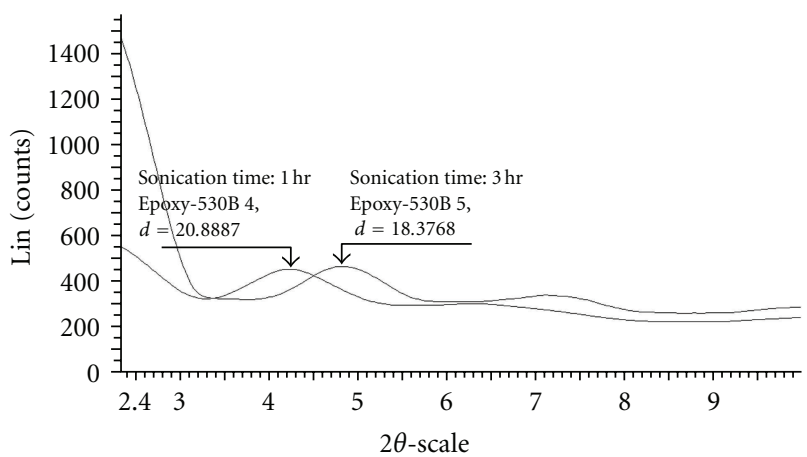

FIGURE 2: XRD spectra of sample prepared with (a) 1 hour and (b) 3-hour sonication treatment.

groups. The increase in molecular weight can also raise the $T_{g}$. A high density of branches and crosslinks can reduce the chain mobility and increase the $T_{g}$ [11].

The inflection slope $\left(m_{g}\right)$ can represent the rate of glass transition process. The author found that this parameter is unlikely being reported in any literature. The lower value of $m_{g}$ may indicate the slower rate of glass transition process,

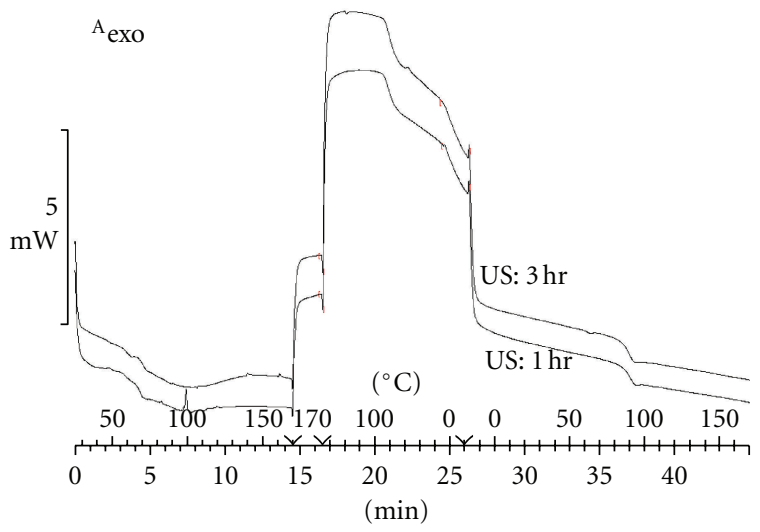

FIGURE 3: The DSC diagram of epoxy clay nanocomposite prepared by mechanical stirring and ultrasonic treatment for 1 and 3-hour duration.

hence shows that the material is thermally more stable upon increased temperature. It seems that 1-hour sonicated processed sample has a lower $m_{g}$.

The specific heat increment value $\left(\Delta c_{g p}\right)$ may represent the increase in rubbery nature of epoxy chain before and after 
TABLE 2: The thermalgravimetric analysis on samples.

\begin{tabular}{lccr}
\hline Sample & $\begin{array}{c}\text { Onset of decomposition } \\
\text { temperature }\left({ }^{\circ} \mathrm{C}\right)\end{array}$ & $\begin{array}{c}\text { Temperature at maximum } \\
\text { decomposition rate }\left({ }^{\circ} \mathrm{C}\right)\end{array}$ & Residual weight $(\%$ wt $)$ \\
\hline Ep 530B US 1 hr & $367 \pm 2.33$ & $378 \pm 6.36$ & $11.3 \pm 0.16$ \\
Ep 530B US 3 hr & $365 \pm 1.06$ & $384 \pm 0.85$ & $12.4 \pm 1.78$ \\
\hline
\end{tabular}

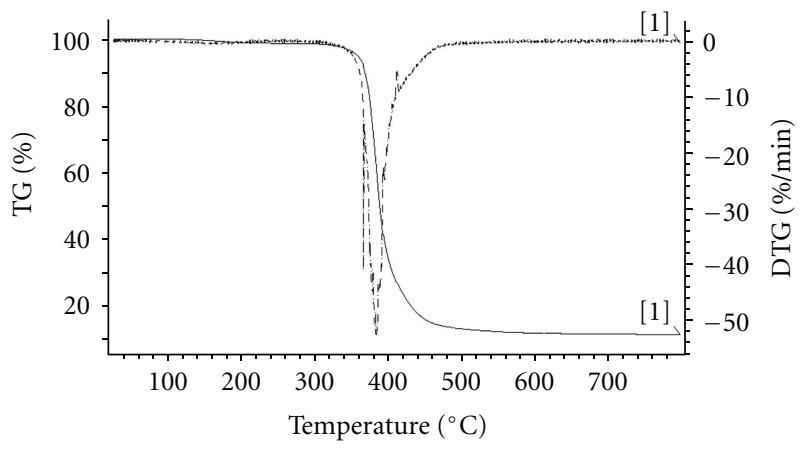

FIGURE 4: TG and DTG of epoxy clay nanocomposite prepared by mechanical stirring and ultrasonic treatment of 1-hour duration.

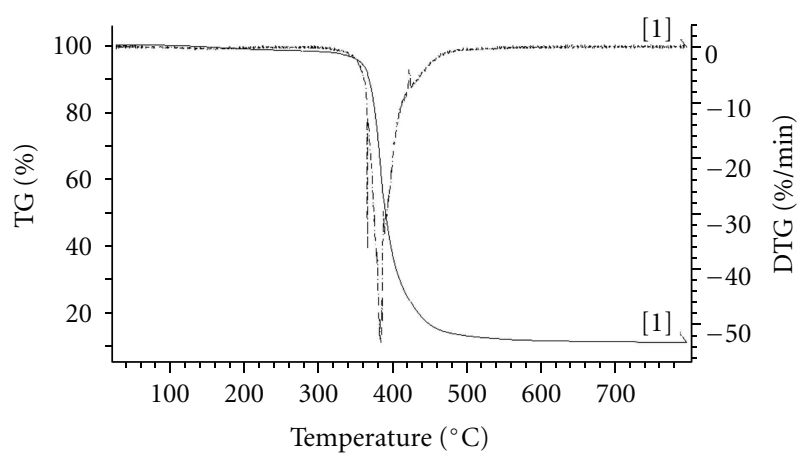

FIGURE 5: TG and DTG of epoxy clay nanocomposite prepared by mechanical stirring and ultrasonic treatment of 3-hour duration.

the glass transition process. Lower values of $\Delta c_{g p}$ indicate more rigid material.

The 1-hour sonicated samples seem to be more rigid and thermally stable during the glass transition process. The 3hour processed sample could have faced degradation of the epoxy matrix upon the longer time of sonication process.

3.4. Thermalgravimetric Analysis. The TGA and DTG diagrams of epoxy nanocomposite samples prepared by 1 - and 3-hour sonicated treatment are given in Figures 4 and 5, respectively. The analysis tabulated in Table 2.

The multiple peaks at DTG graph, may indicate decomposition of different molecular weight network of epoxy. Furthermore, the presence of clay can disrupt the decomposition process.

The onset of decomposition temperatures for both samples are about $366^{\circ} \mathrm{C}$. It appears that the duration of ultrasonic treatment does not affect the onset decomposition temperature. However, the maximum decomposition rate occurred at higher temperature for 3-hour sonicated samples, indicating that it is more thermally-stable during decomposition process. This may be due to the presence of higher crosslink density and better clay dispersion. The residual weight for both samples is quite consistent, at about $12 \mathrm{wt} \%$.

\section{Summary}

The ability of ultrasonic treatment to separate individual silicate in viscous epoxy mixtures was proven, by TEM and XRD analysis. However, longer duration of ultrasonic treatment may not assist the silicate separation, due to the increased viscosity of the mixture during the process. The 1-hour sonicated processed sample possessed higher glass transition temperature, but less thermally stable during decomposition process, compared to the one prepared by 3-hour sonicated process. This may be attributed to the differences in clay dispersion trends, crosslink density, and voids presence inside the sample.

\section{Acknowledgments}

The authors would like to acknowledge help from Ms. Nor Maizana Zakaria from Rubber Research Institute of Malaysia, Ms. Suhaniza Razali, Mr. Ahmad Zaki Zaini, Mr. Zailan Mohd. Yusof, Mr. Wan Mohd Nazir Bin Wan Taha, and Mr. Mohd. Razif Maafol for their kind assistance. Also, the authors wish to acknowledge Universiti Kebangsaan Malaysia, Malaysia Public Service Department (PSD), and the Ministry of Science, Technology and Innovation (MOSTI) for SLAI scholarship and Science Fund Grant (03-01-02-SF0059) to conduct the research.

\section{References}

[1] J. G. Ryu, H. Kim, and J. W. Lee, "Polymer-clay nanocomposites prepared by power ultrasonic wave; synthesis and rheological properties," in Proceedings of the PPS-18 Annual Meeting, p. 100, 2002.

[2] G. Camino, G. Tartaglione, A. Frache, C. Manferti, and G. Costa, "Thermal and combustion behaviour of layered silicate-epoxy nanocomposites," Polymer Degradation and Stability, vol. 90, no. 2, pp. 354-362, 2005.

[3] Y. Zhou, F. Pervin, M. A. Biswas, V. K. Rangari, and S. Jeelani, "Fabrication and characterization of montmorillonite clayfilled SC-15 epoxy," Materials Letters, vol. 60, no. 7, pp. 869$873,2006$.

[4] G. Bernard, R. D. West, and V. M. Malhotra, "Mechanical behavior of cured epoxy: effects of ultrasonic processing," in Proceedings of the Society of Plastics Engineers Annual Technical Conference (ANTEC '06), pp. 1702-1706, usa, May 2006. 
[5] O. Becker and G. P. Simon, "Epoxy layered silicate nanocomposites," Advances in Polymer Science, vol. 179, pp. 29-82, 2005.

[6] O. Becker, G. P. Simon, R. J. Varley, and P. J. Halley, "Layered silicate nanocomposites based on various high-functionality epoxy resins: the influence of an organoclay on resin cure," Polymer Engineering and Science, vol. 43, no. 4, pp. 850-862, 2003.

[7] J. Fröhlich, R. Thomann, O. Gryshchuk, J. Karger-Kocsis, and R. Mülhaupt, "High-performance epoxy hybrid nanocomposites containing organophilic layered silicates and compatibilized liquid rubber," Journal of Applied Polymer Science, vol. 92, no. 5, pp. 3088-3096, 2004.

[8] A. J. Kinloch and A. C. Taylor, "Mechanical and fracture properties of epoxy/inorganic micro- and nano-composites," Journal of Materials Science Letters, vol. 22, no. 20, pp. 1439-1441, 2003.

[9] C. L. Chiang, R. C. Chang, and Y. C. Chiu, "Thermal stability and degradation kinetics of novel organic/inorganic epoxy hybrid containing nitrogen/silicon/phosphorus by sol-gel method," Thermochimica Acta, vol. 453, no. 2, pp. 97-104, 2007.

[10] N. Y. Yuhana et al., "Postcuring effect on the glass transition temperatures of PMMA grafted natural rubber toughened epoxy layered silicate nanocomposite".

[11] W. D. Callister, Material Science and Engineering: An Introduction, John Wiley \& Sons, 7th edition, 2007. 

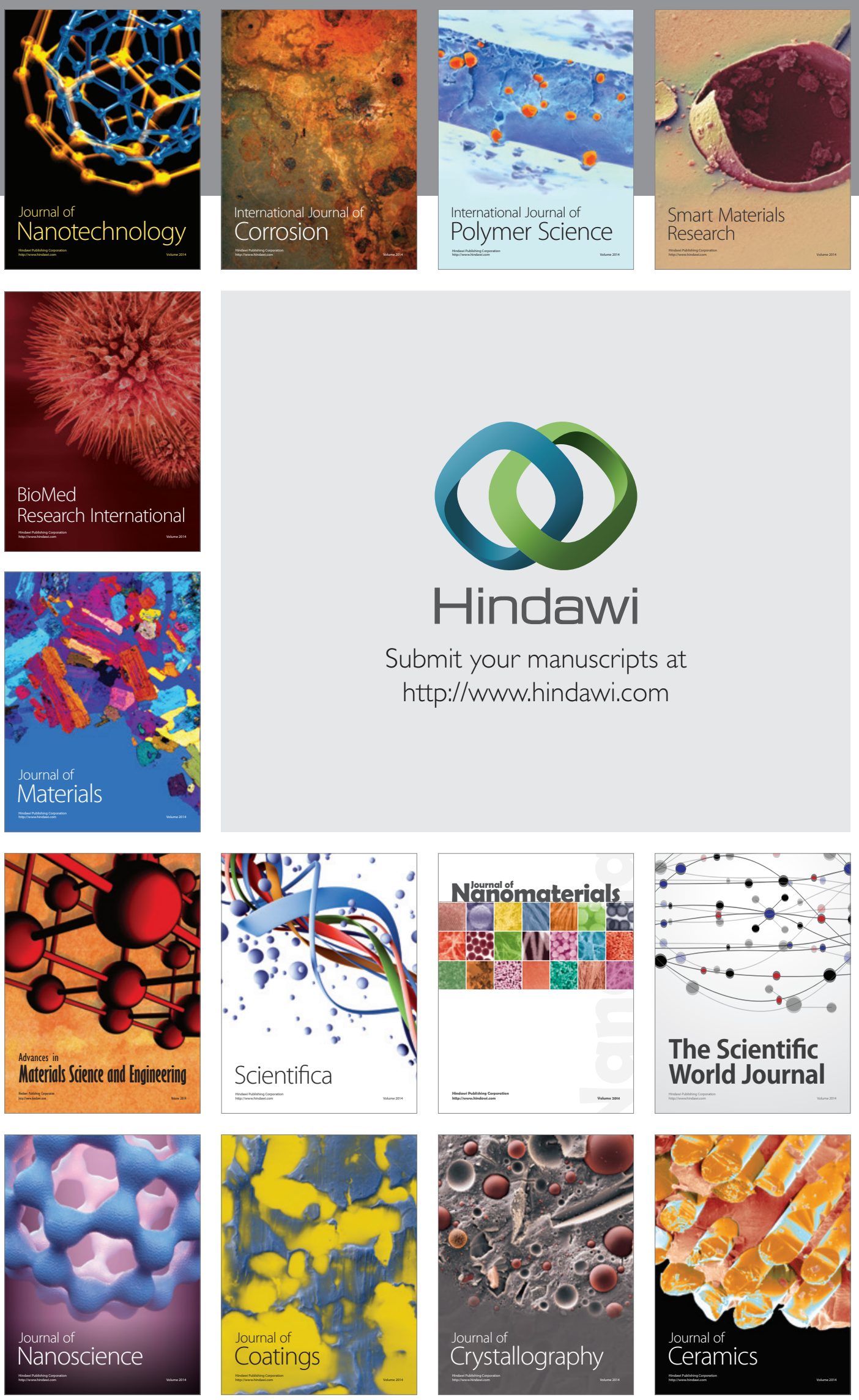

The Scientific World Journal

Submit your manuscripts at

http://www.hindawi.com

\section{World Journal}

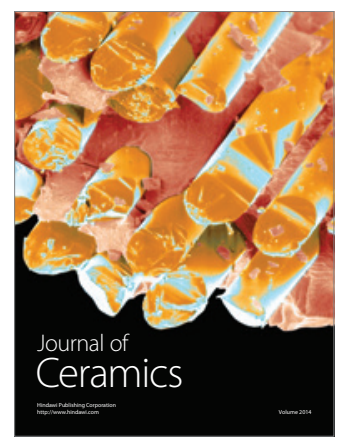

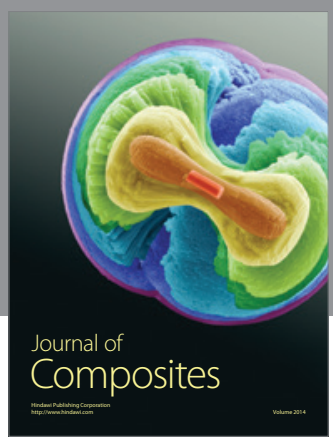
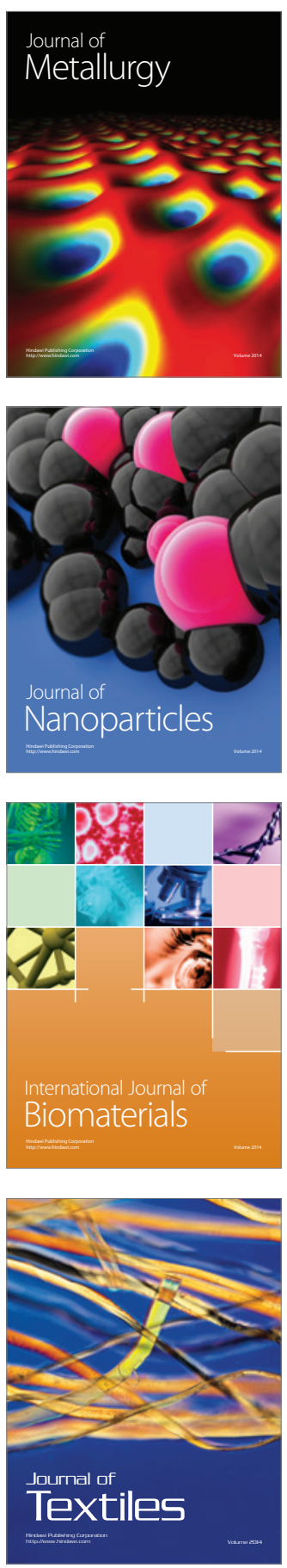